\title{
Remote Sensing for Medical and Health Care Applications
}

\author{
Satoshi Suzuki and Takemi Matsui \\ Kansai University, Tokyo Metropolitan University \\ Japan
}

\section{Introduction}

Radar-based remote sensing techniques are typically employed to determine the velocities and positions of targets such as aircraft, ships, and land vehicles. In particular, X- and Kband microwave devices, including oscillators and antennas, have been used to measure the velocity of automobiles and other moving objects in recent years. Microwave devices that are compact, accurate, reliable, and inexpensive are currently commercially available. Over the past few years, there have been increasing attempts to apply such techniques to biomedical measurements. Although some studies have applied these devices to medicine and health care, such research is still in its infancy. This chapter focuses on the mechanisms of and the recent research trends in microwave remote sensing techniques that are used to detect minute vibrations on the body surface induced by heartbeat and respiration.

\subsection{Background}

The increasing proportion of elderly in the population represents an appreciable problem in developed countries due to social concerns such as increased medical and social welfare costs and a shortage of manpower. Such concerns are expected to worsen in the future. It is thus necessary to focus on preventing illnesses and to promote healthy lifestyles. Consequently, simple equipment that can be used to self-monitor medical conditions and to acquire related data is required for homes as well as medical facilities.

Vital signs are parameters of physiological functions that are used to express the physical condition. They are used by medical professionals for making initial diagnoses. There are four primary vital signs: heart rate, respiratory rate, body temperature, and blood pressure. Thermometers for home use are commercially available and are generally approved by medical bodies. In addition, heart rate and respiratory rate can be easily confirmed by visual and palpation methods. However, there is currently still not spread to home device capable of accurately measuring and recording vital sign data that can be used to make detailed diagnoses. Monitoring cardiac function can be used for diagnosing arrhythmia and mental stress (Akselrod et al., 1981, Singh et al., 1996, Carney et al., 2001). Recently, monitoring mental condition has attracted more attention than monitoring physiological parameters. And also obesity and aging are thought to contribute to the risk of developing sleep apnea 
syndrome (SAS). Airway obstruction due to fat deposition in the neck is one cause of SAS and it is related to reduced alertness during the daytime (Morriset al., 2008). A simple device that can monitor respiratory activity throughout the night is thus required. These examples show the necessity for monitoring of the vital signs in daily life. Moreover, these sensing technique are presently being studied in the research area on human-machine interfaces that can be applied anywhere (for example, in a car or at the workplace) (Sirevaag et al., 1993, Gould et al., 2009).

In addition, patients who have been exposed to toxic chemicals or infectious diseases are often treated in isolation chambers to prevent secondary exposure to health-care workers. In such cases, a doctor must often make a diagnosis without touching the patient, which is difficult as the vital signs are of primary importance for emergency medical treatment. With the exception of body temperature (which can be measured by infrared radiation), it is difficult to measure vital sign parameters without contact. Consequently, remote sensing of vital signs has attracted much attention.

In this way, several fields require remote sensing of vital signs and various remote sensing methods have been proposed. However, such methods should perform biomedical measurements described as non-invasive, non-restrictive, or non-contact means that can be used without the user being conscious of them. The use of physically attaching sensors should be minimized to reduce the burden on users.

\subsection{Purpose and requirements of remote sensing in medicine and health care}

Monitoring cardiac and respiratory parameters is useful for health-care management as users go about their everyday lives. However, such daily monitoring needs to overcome many problems. For example, users must have sufficient technical and medical competence to set electrodes to themselves and they must not feel physically restricted by the electrodes and leads. To overcome such problems, research is increasingly being conducted on developing non-invasive and non-restrictive sensing techniques for acquiring vital signs (Jacobs et al., 2004, Wang et al., 2006, Ciaccio et al., 2007). This kind of sensing technique aims to detect and measure vibrations on the body surface induced by cardiac and respiratory activity. In the case of respiratory activity, a person's abdomen expands and contracts during the breathing cycle and this movement can be detected by sensing techniques. Similarly, for cardiac activity, the body surface moves in response to the heartbeat in minute scales. Although the vibration is slight and its amplitude depends on the individual and the part of the body, it has been observed from all parts of the body with an average amplitude of about $0.1-0.2 \mathrm{~mm}$ by a high-resolution laser distance meter (Suzuki et al., 2011).

Some studies have measured heart rate by placing a pressure sensor (Jacobs et al., 2004) or polyvinylidene fluoride piezoelectric sensors (Wang et al., 2006) between the user and the mattress on which they sleep. This kind of measurement method measures responses to pressure changes. Other trials have used strain gauges to measure the heart rate (Ciaccio et al., 2007). The size of minute changes due to pressure changes on the body surface induced by the heartbeat and information relating to heartbeat and respiration were obtained. A similar procedure was employed in studies using air mattresses (Watanabe et al., 2005). 
Such sensing techniques have the advantages of being inexpensive because of their simple structure and of enabling stable relatively stable data acquisition because they employ direct contact with the body. Some of these sensors are already commercially available. However, they suffer from one drawback: measurement is not possible when the sensor is separated from the body by moving their bodies. This raises the question: "Is a remote sensing method available?"

\subsection{Biomedical measurement using microwaves}

Radio-frequency sensing techniques were originally developed for military applications and they were used to determine the location and velocity of aircraft and ships. The same technology was then applied to search and rescue; for example, they have been used to locate survivors buried under earthquake rubble (Chen et al., 1986, 2000, Lin et al., 1992). Radar can remotely acquire information on the motion of targets. Additionally, depending on the frequency of the electromagnetic wave used, radar can penetrate barriers. These characteristics of radar have been employed to detect body motion of survivors under earthquake rubble. Such devices initially had very limited effectiveness because of their poor resolution by using low-frequency waves to penetrate rubble; they could only detect relatively large body motion (at best, the abdominal motion due to breathing). However, the permeability is not a problem for everyday applications since microwaves can readily penetrate materials such as clothing, bedding, and mattresses.

A cheap, small unit that is stable and can oscillate at high frequencies has recently been developed and ongoing development is being conducted to produce safer, more flexible devices. As a consequence, higher frequency electromagnetic waves were contributed to enhance the resolution of measurement. At the same time, the output power was reduced to reduce its effect on humans, allowing microwaves to be used in everyday applications. Gradual progress, therfore, has made it possible to detect even human heartbeat.

\section{Theory and methods}

Here, we describe a system that employs microwaves to remotely measure vital signs by detecting vibrations on the body surface induced by cardiac and respiratory activity. Vibrations induced by heartbeat are particularly small with amplitudes of about $0.1-0.2 \mathrm{~mm}$ on average. This section discusses approaches using continuous-wave (CW) Doppler radar and ultra-wideband (UWB) pulse radar, which are generally used for measuring vital signs, and their mechanisms.

\subsection{Mechanisms of measurement}

While frequency-modulated continuous wave (FMCW) radar is used to identify the exact location of a subject in some reports, UWB or CW Doppler radar are generally used for monitoring vital signs. (Saunders, 1990, Immoreev \& Tao, 2008, Li \& Lin, 2010)

In a UWB pulse radar, the transmitter sends very short electromagnetic pulses toward the target. A pulse duration of about $200-300$ ps and a pulse repetition frequency in the range of 1-10 MHz are typically used for vital sign detection. When the transmitted pulse reaches the chest wall, some of the energy is reflected and captured by the receiver. The nominal round- 
trip travel time of the pulse is defined as $t=2 d / C$, where $\mathrm{d}$ is the nominal detection distance and $C$ is the speed of the electromagnetic wave. If a local replica of the transmitted pulse with a delay close to the nominal round-trip travel time correlates with the received echo, the output correlation function will have the same frequency as the physiological movement.

On the other hand, the CW Doppler radar mechanism is based on following (1);

$$
T(t)=\cos [2 \pi f t+\varphi(t)]
$$

where an unmodulated signal $T(t)$ with a carrier frequency $f$ and a residual phase $\varphi(t)$, is transmitted toward a human body where it is phase-modulated by the physiological movement $x(t)$. The reflected signal $R(t)$ detected by the radar receiver is given by following (2);

$$
R(t) \approx \cos \left[2 \pi f t-\frac{4 \pi d_{0}}{\lambda}-\frac{4 \pi x(t)}{\lambda}+\varphi\left(t-\frac{2 d_{0}}{c}\right)\right]
$$

where $4 \pi d_{0} / \lambda$ is a constant phase shift due to the nominal detection distance $d_{0}$ and the $\varphi\left(t-2 d_{0} / c\right)$ is phase noise. Using the same transmitted signal $T(t)$ as the local oscillator signal, the radar receiver down-converts the received signal $R(t)$ to the baseband signal $B(t)$ as following (3);

$$
B(t) \approx \cos \left[\frac{4 \pi d_{0}}{\lambda}+\frac{4 \pi x(t)}{\lambda}+\theta_{0}+\Delta \varphi\right]
$$

where $\Delta \varphi$ is determined by the nominal detection distance and the oscillator phase noise.

Since the delay corresponds to the signal round-trip travel time, the detection range of a UWB radar can be varied by controlling the delay between the two inputs of the correlation function block. This makes it possible to eliminate interference caused by reflection from other objects (clutter) and multipath reflection. However, one disadvantage of UWB radar is that the delay needs to be recalibrated when the detection distance is changed; this increases the system complexity and cost. Furthermore, since the correlation function is nonlinear, it is not simple to recover the original movement pattern, even though frequency information can be easily obtained. On the other hand, CW Doppler radar has a low power consumption and a simple radio architecture. These characteristics make it suitable for home-based systems. Moreover, proper adjustment of the radio front-end architecture of a CW radar can cancel clutter (Li \& Lin, 2008a, 2008b). In addition, single-input multi-output and multiinput multi-output techniques can be easily implemented with CW radar, enabling the movements of multiple targets to be detected (Boric-Lubecke et al., 2005, Zhou et al., 2006).

\subsection{Carrier frequency and output power}

The carrier frequency and output power employed must be safe for use on people. Carrier frequencies ranging from hundreds of megahertz to millimeter wave frequencies have been tested for remote vital sign detection using a variety of physiological movements. The carrier frequency should be carefully selected to ensure suitable sensitivities and 
characteristic response for vital sign measurement. Some studies used extremely highfrequency waves (228 GHz (Petkie et al., 2009)), which have shorter wavelengths and are more sensitive to small displacements. Moreover, a $228 \mathrm{GHz}$ frequency prototype has been extended to perform heart rate and respiration measurements at a distance of $50 \mathrm{~m}$. However, such high frequency waves are not realistic for monitoring vital signs in everyday applications. In many cases, a carrier frequency that does not require a license is often chosen. However, carrier frequencies that do not require a license vary from country to country and some frequency bands are allocated to amateur radio stations. For example, the laws regulating radio frequency use in Japan allow band frequencies 10.525 and $24.15 \mathrm{GHz}$ to be used for detecting moving objects. These devices are marketed as sensors for measuring the speeds of vehicles. Although there are limitations on how they are used (e.g., limited to indoor use), these frequency bands can be used by low-power radio stations without a license provided the output is less than $10 \mathrm{~mW}$. They have been increasing studies on frequency bands for vital sign monitoring. Regarding safety, different countries have different guidelines regarding radio-frequency electromagnetic fields.

The World Health Organization (WHO) and the Scientific Committee on Emerging and Newly Identified Health Risks (SCENIHR) define exposure as the subjection of a person to electric, magnetic, or electromagnetic fields or to contact currents other than those originating from physiological processes in the body and other natural phenomena (WHO, 2003, SCENIHR, 2006). The intended frequency band of electromagnetic field intensity differ slightly in different guidelines. For example, the International Commission on Non-Ionizing Radiation Protection (ICNIRP) guidelines (ICNIRP, 1998) specify the frequency band from $300 \mathrm{~Hz}$ to $300 \mathrm{GHz}$, SCENIHR specifies $100 \mathrm{~Hz}$ to $300 \mathrm{GHz}$ (SCENIHR, 2006), and the IEEE Standard is from $3 \mathrm{kHz}$ to $300 \mathrm{GHz}$ (IEEE Standard Committee, 1998). Each country employs different methods for determining their criteria. Consequently, it is important consider device development and intended usage.

The carrier frequency of medical applications of ultrasound is a low frequency of about 3 to $10 \mathrm{MHz}$. Such applications acquire information by penetrating the human body. In comparison, monitoring using microwave frequencies in the range 10.525 to $24.15 \mathrm{GHz}$ is considered to be less invasive and safer. It is not easy to make simple comparisons, but wireless local area networks (WLANs) use $2.4 \mathrm{GHz}$ radio waves and microwaves in the range 10.525 and $24.15 \mathrm{GHz}$ are considered to be safer. Moreover, safety can be further increased by using a lower power than a WLAN.

It seems appropriate to use high frequencies for sensing to ensure a high resolution while considering invasiveness. However, high frequencies are not necessarily ideal for actual applications as increased sensitivity results in increased susceptibility to artifacts; the target motion induced by heartbeat on the body surface is much smaller than the artifacts generated by general movement of the body and arm. Furthermore, people being monitored move freely as they conduct everyday activities, which makes artifacts a significant problem.

\section{Examples of applications}

While remote sensing is not currently used for medical and health care applications in everyday life, several studies have been conducted. This section discusses the following 
typical examples of remote sensing in medical and health care settings: (1) monitoring daily health and mental stress by estimating changes in the autonomic nervous system (ANS); (2) welfare and health care for the elderly; and (3) medical diagnosis such as screening of patients with infections.

\subsection{Measurement of ANS}

To determine stress levels when driving or operating equipment, a $24 \mathrm{GHz}$ compact microwave radar was used to perform remote measurements of the heart rate variability (HRV) under autonomic activation induced by a stressful sound and foods (Suzuki et al., 2008, Gotoh et al., 2009). Changes in the ANS (sympathovagal balance) can be monitored by measuring the HRV. This variability is used as an index of mental stress.

For examples, when an animal is attacked, its sympathetic nervous system spontaneously prepares for fight or flight by elevating the heart rate and increasing the blood pressure and body temperature. In contrast, when it is in a relaxed state, the parasympathetic nervous system is activated, and the opposite phenomena occur. Such processes are constantly in equilibrium in daily life (Akselrod et al., 1981, Derrick,1988).

Sympathovagal balance can be monitored by measuring variations in the heartbeat interval, and the degree of mental stress can be determined. This index is used for diagnosing psychological disorders and as well as the condition of circulatory organs. Moreover, many approaches for measuring the HRV are used in psychology (Vincent et al., 1996), occupational health (Miyake, 2001, Princi et al., 2005), and ergonomics research (Sirevaag et al., 1993, Gould et al., 2009). An estimation technique has been medically established. Thus, if electrocardiograms (ECG) can be replaced by a remote sensing method that allows simple and accurate monitoring in everyday life, mental stress can be assessed at any time and in any place.

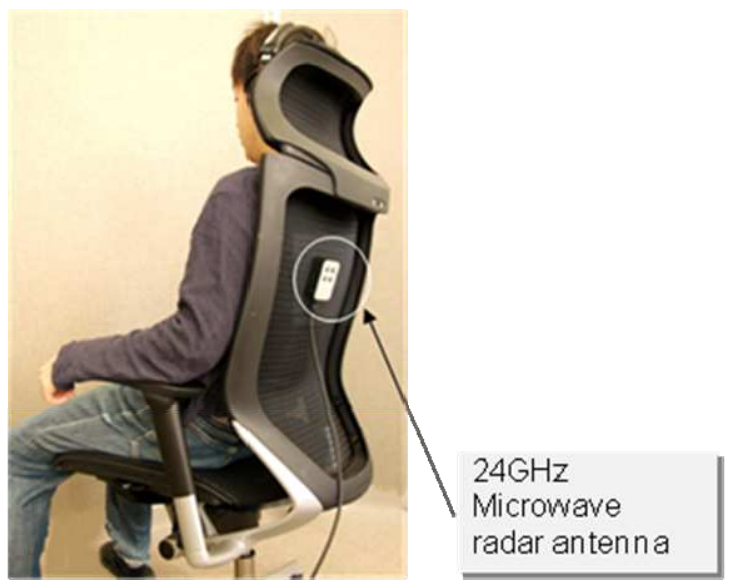

Fig. 1. Prototype chair equipped with a non-contact microwave radar system to monitor mental stress in workers (Suzuki et al., 2008). 


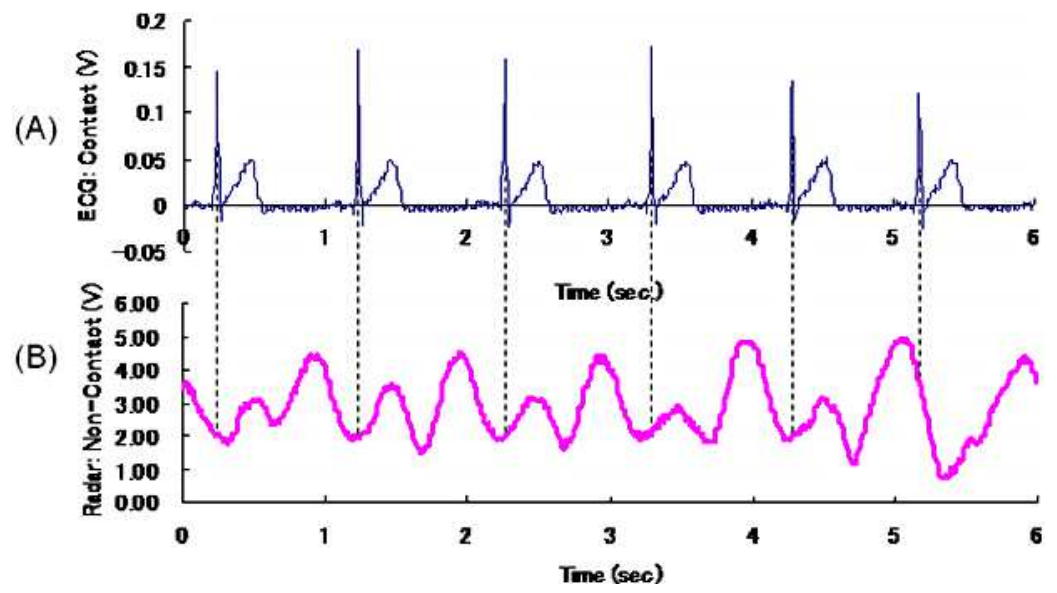

Fig. 2. Sample data of a compact microwave radar output (A) showing a cyclic oscillation that corresponds to cardiac oscillations measured by ECG (B) (Suzuki et al., 2008).

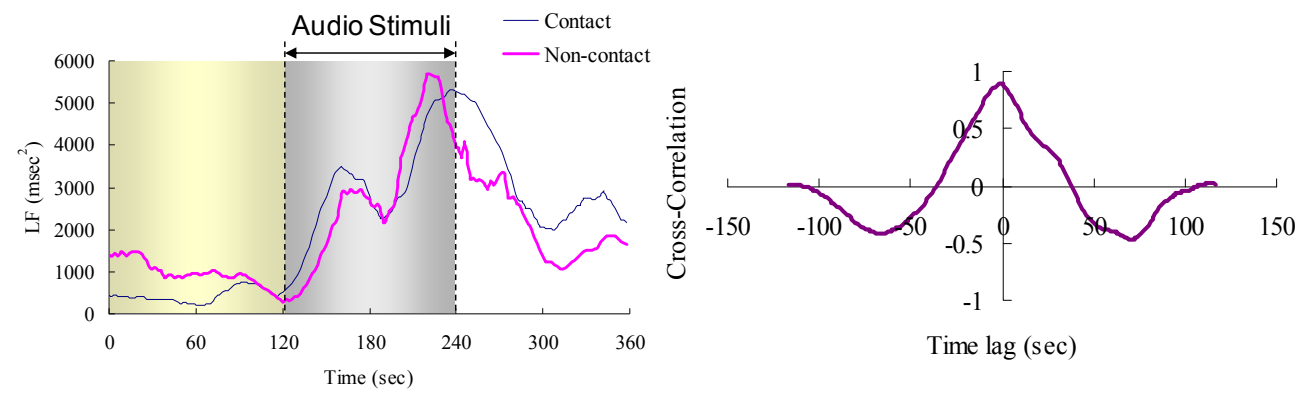

(A)

(B)

Fig. 3. Example of remote sensing in health care. (A) In both non-contact and contact (ECG) measurements, the LF of a subject (reflecting sympathetic activation) exhibits a peak during audio stimulation. (B) Cross-correlation of the LF between non-contact and contact measurements of the same subject (Suzuki et al., 2008).

The prototype device in this example consists of a compact $24 \mathrm{GHz}$ microwave Doppler radar $(8 \times 5 \times 3 \mathrm{~cm})$ attached to the back of a chair. The low-frequency/high-frequency (LF/HF) components of the HRV (which reflects the sympathovagal balance) of eight volunteers were determined by a prototype system using the maximum entropy method (MEM). The change in the LF and LF/HF components measured by a remote sensor was consistent with that measured by a contact electrocardiography sensor. It is very difficult to extract R-R intervals of heartbeats sufficiently accurately to calculate the HRV; this is thought to be because inaccuracies were introduced due to the microwave radar being susceptible to noise. However, measuring HRV by adopting MEM, which enables HRV to be estimated rapidly, stably, and accurately, was demonstrated to be successful for the first time. 


\subsection{Elderly care}

The next examples were monitoring of the elderly, infants and also animals using a microwave radar, especially focused respiratory monitoring ( $\mathrm{Li}$ et al., 2009, Suzuki et al.,2009).

There is a real need to reduce the physical and mental work load of care workers and also to immediately detect sudden changes in the condition of a bedridden elderly person, both at home and in hospitals, because nighttime activity of persons with dementia increases the risk of injury and disrupts the sleep patterns of caregivers. Moreover, care of the elderly will increasingly be undertaken by family members at home as the population continues to age. Therefore, devices for long-term monitoring of the elderly that do not interfere with activities of daily life are required.

Various approaches have been proposed in recent years. Rowe (Rowe et al., 2009) proposed a night monitoring system that alerts caregivers when care recipients leave their beds and that tracks them as they move about the house during the night. In addition, another study compared the effects of a self-care and medication compliance device, which was linked to a web-based monitoring system, with the effects of conventional care alone on compliance with recommended self-care behavior (Artinian et al., 2003). Demiris (Demiris et al., 2009) investigated elderly behavior using a video and image processing system while carefully addressing privacy concerns. Another study proposed a non-restrictive, non-invasive vital sign measurement system for measuring heartbeat and respiration to monitor health status at home or in hospitals and nursing facilities (Tanaka et al., 2002). Systems for nursing care should meet the following requirements: (1) monitoring of activity and vital signs must not be a burden for the elderly; (2) accurately monitor changes in physical condition of the patient; and (3) when the physical condition of a patient rapidly changes, the system should promptly notify a doctor or nursing caregiver.

A prototype system using microwaves has been developed and has been applied for monitoring elderly in a nursing home. Although still a trial, the system can effectively perform real-time monitoring and it can acquire SAS respiratory data.

In addition, a baby also monitor using same technology has recently been demonstrated (Li et al., 2009). The baby monitor integrates a low-power Doppler radar that can detect minute movements induced by breathing. If no movement is detected within $20 \mathrm{~s}$, an alarm goes off.

Using same type of sensing devices, respiratory activity of a hibernating black bear has been monitored at a Zoo (Suzuki et al., 2009). Ueno Zoological Gardens in Tokyo made plans to assist a Japanese black bear to enter hibernation, because the bear showed extremely slow movements in winter. Moreover, the staff wanted visitors to understand this instinctive behavior of bears as occurs in the wild. The bear's condition during hibernation must be carefully observed to avoid the risk of long-term fasting. Therefore, to observe the physiological condition of the bear during hibernation, about 3 months, a microwave radar system was set up in the hibernation booth. As a result, the respiratory rate decreased while the bear was entering hibernation, and became extremely low at approximately $2 \mathrm{bpm}$ and showed almost no change. Additionally, a trend similar to a circadian rhythm in the changes of respiratory rate was observed. 


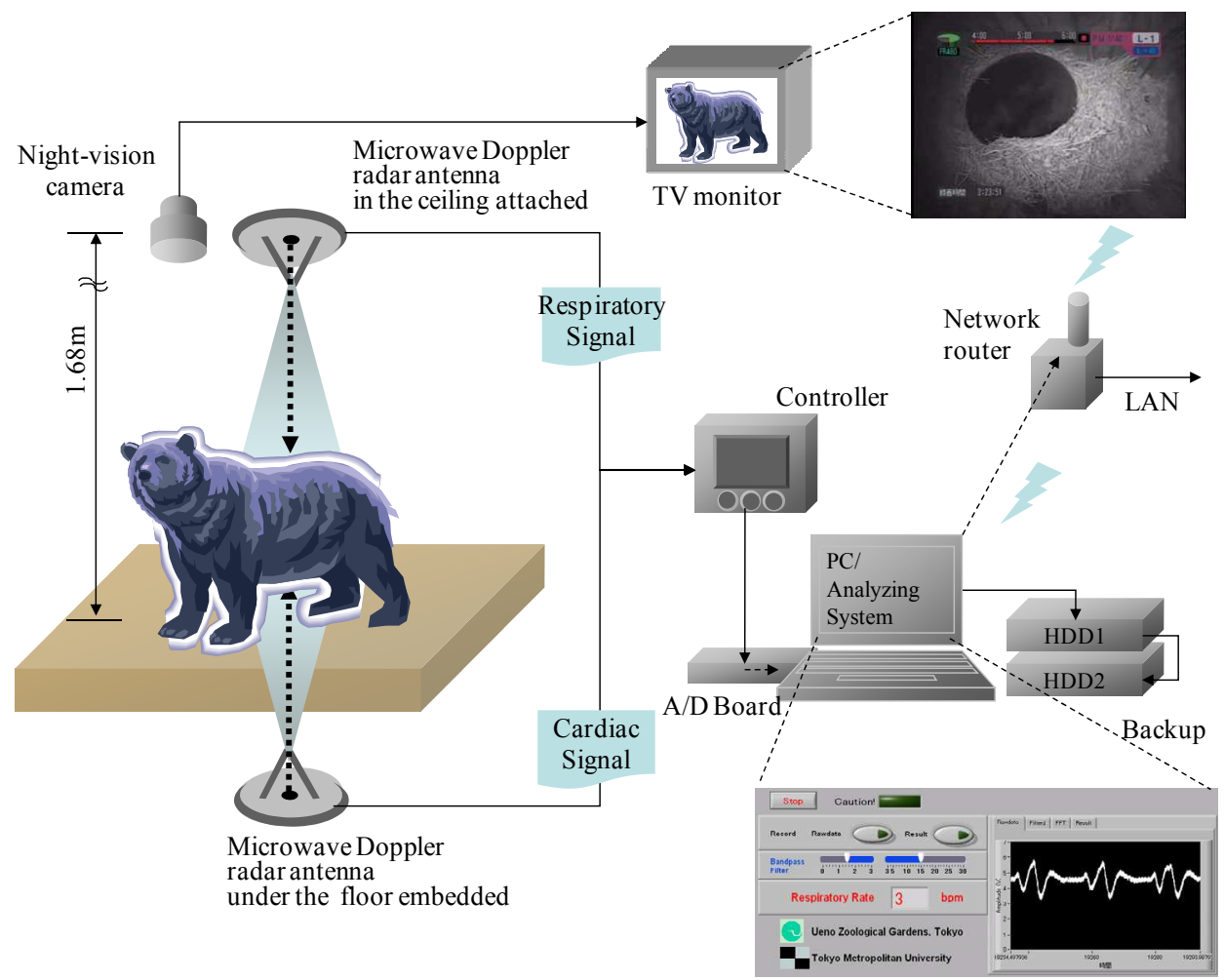

Fig. 4. Example of remote sensing using non-contact vital sign sensors to monitor a hibernating black bear at Ueno Zoo, Tokyo (Suzuki et al., 2009).

\subsection{Screening and medical examination of influenza patients}

The advantage of monitoring without direct touch and without removing clothing is useful and appealing in the medical field as it is not burdensome to patients and it can reduce the risk of secondary infection. There is also growing interest in the fields of health, life science, and engineering.

In another trial, a new screening system was developed to conduct rapid screening $(<5 \mathrm{~s})$ of passengers who may have infectious diseases such as severe acute respiratory syndrome (SARS) or pandemic influenza at quarantine stations. This system enables medical inspection by measuring heart and respiratory rates, as well as body temperature by infrared thermography (Matsui et al., 2009, 2010). A similar system is already being tested at the quarantine station at Naha Airport for domestic flights and at Narita International Airport in Tokyo. The results demonstrate the efficacy of the concept and the system. The system even detected a patient with influenza whose fever was reduced by antifebrile medication. This finding is important as it demonstrates that the parameters 
for monitoring cardiac and respiratory activity by microwaves are effective. In the future, remote sensing using microwaves is anticipated to attract more interest in the medical field.

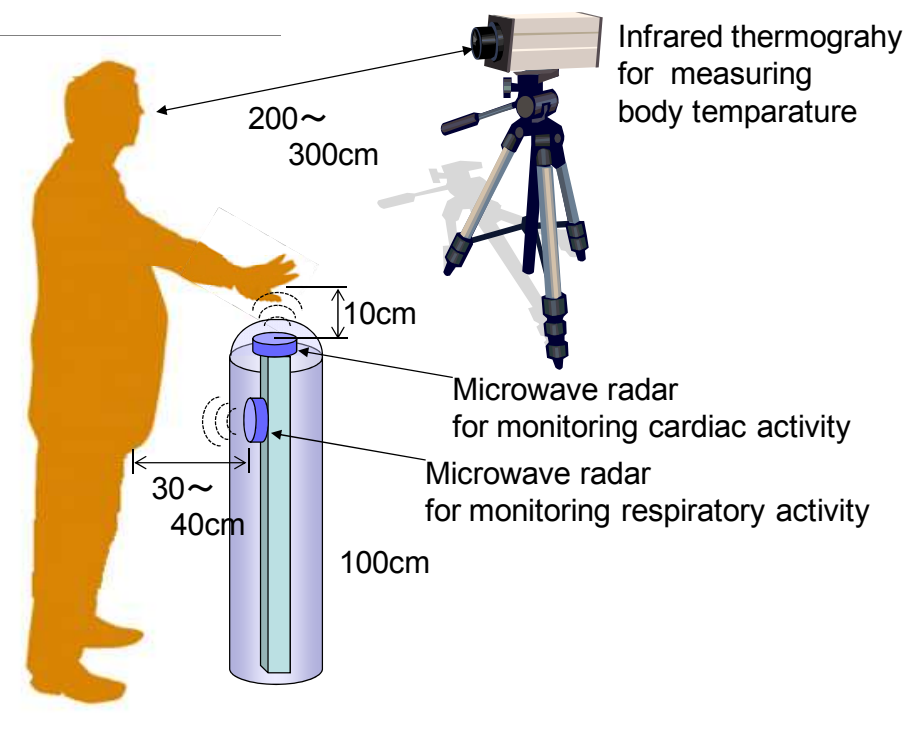

Fig. 5. Example of remote sensing for medical diagnosis; non-contact monitoring for screening systems at airport quarantine stations (Matsui et al., 2009).

\section{Topics for future investigation}

Remote sensing of biomedical parameters has been described by focusing on monitoring using microwave radar and examples of medical and health care applications have been presented. Microwave-based remote sensing offers the following advantages: movement of an object can be detected from a distance, it means "remotely", and microwaves can pass through many materials (notable exceptions are metals and water). Thus, such systems will enable health care workers to measure motion of a body surface through clothing from a distance.

The most serious obstacle to practical applications of this technology is the need to reduce the effects of noise and artifacts. Vibrations on the body surface caused by heartbeat are quite small (with amplitudes of about 0.1-0.2 mm), whereas motions of arms and the abdomen are much larger. Additionally, movement of the person being monitored while conducting every day activities and data from other body motion will generate noise. The effect of multipass reflection should also be considered. One study performed simultaneous measurements of the vital signs of two people, but it is difficult to apply this technology to real-world applications. 
To use this technique in medical fields and daily life, aspects such as non-contact measurement and the non-removal of clothing need to be addressed. Data obtained by remote sensing must be more reliable and accurate than those measured by conventional electrode-based methods. Furthermore, a new index for diagnosing disease is required. Most studies of remote sensing using microwaves only measure the heart rate. However, the heart rate alone has limited diagnostic value; the heart rate only provides information about the tachycardia or bradycardia. It is hoped that such a system will be able to measure parameters for diagnosing cardiovascular disease.

In conclusion, research on remote sensing using microwaves is still in its infancy, but it appears promising not only for medical and health care, but also for other fields such as ergonomics research.

\section{References}

Akselrod, S. et al. (1981) Power spectrum analysis of heart rate fluctuation: a quantitative probe of beat-to-beat cardiovascular control. Science, 213(4504), pp.220-222.

Artinian, N.T., Harden, J.K., Kronenberg, M.W., Vander Wal, J.S., Daher, E., Stephens, Q., Bazzi, R.I. (2003) Pilot study of a Web-based compliance monitoring device for patients with congestive heart failure. Heart Lung, 32(4), pp.226-233.

Boric-Lubecke, O., Lubecke, V., Host-Madsen, A., Samardzija, D., Cheung, K. (2005) Doppler radar sensing of multiple subjects in single and multiple antenna systems. 7th International Conf. on Telecom. In Modern Satellite, Cable and Broadcasting Services, 1, pp. 7-11.

Carney, R.M., Blumenthal, J.A., Stein, P.K., Watkins, L., Catellier, D., Berkman, L.F., Czajkowski, S.M., O'Connor, C., Stone, P.H., Freedland, K.E. (2001) Depression, heart rate variability, and acute myocardial infarction. Circulation, 104, pp.20242028.

Chen, K.M., Misra, D., Wang, H., Chuang, H.R., Postow, E. (1986) An X-band microwave life-detection system. IEEE Trans Biomed Eng, 33, pp.697-702.

Chen, K.M., Huang, Y., Zhang, J. (2000) Microwave Life-Detection Systems for Searching Human Subjects Under Earthquake Rubble or Behind Barrier. IEEE Trans Biomed Eng, 27, pp.105-113.

Ciaccio, E.J., Hiatt, M., Hegyi, T., Drzewiecki, G.M. (2007) Measurement and monitoring of electrocardiogram belt tension in premature infants for assessment of respiratory function. Biomed Eng Online 6, pp.1-11

Demiris, G., Oliver, D.P., Giger, J., Skubic, M., Rantz, M. (2009) Older adults' privacy considerations for vision based recognition methods of eldercare applications. Technol Health Care, 17(1), pp.41-48.

Derrick, W.L. (1988) Dimensions of operator workload. Human Factors, 30, pp.95-110.

Gotoh, S., Suzuki, S., Kagawa, M., Badarch, Z., Matsui, T. (2009) Non-contact determination of parasympathetic activation induced by a full stomach using microwave radar, Medical \& Biological Engineering \& Computing, 47, pp.1017-1019. 
Gould, K.S., Roed, B.K., Saus, E.R., Koefoed, V.F., Bridger, R.S., Moen, B.E. (2009) Effects of navigation method on workload and performance in simulated high-speed ship navigation. Appl Ergon 40, pp.103-114.

ICNIRP Guidelines (1998) For Limiting Exposure to Time-Varying Electric, Magnetic and electromagnetic Fields (UP TO 300 GHZ), Health Physics, 74(4), pp.494-522.

IEEE Standards Coordinating Committee 28 on Non-Ionizing Radiation Hazards (1998) IEEE Standard for Safety Levels With Respect to Human Exposure to Radio Frequency Electromagnetic Fields, 3 kHz to 300 GHz. C95.1-1991 and C95.1a-1998.

Immoreev, I., Tao, T.-H. (2008) UWB radar for patient monitoring. IEEE Aerospace and Electronic Systems Magazine, 23(11), pp.11-18.

International EMF Project of World Health Organization (2003) Framework for Developing EMF Standards.

Jacobs, J., Embree, P., Glei, M., Christensen, S., Sullivan, P. (2004) Characterization of a novel heart and respiratory rate sensor. Conf Proc IEEE Eng Med Biol Soc, 3, pp.22232226.

Li, C., Lin, J. (2008) Complex Signal Demodulation and Random Body Movement Cancellation Techniques for Non-contact Vital Sign Detection. IEEE MTT-S International Microwave Symposium Digest, pp.567-570.

Li, C., Lin, J. (2008) Random Body Movement Cancellation in Doppler Radar Vital Sign Detection. IEEE Transactions on Microwave Theory and Techniques, 56(12), pp.3143-3152.

Li, C., Cummings, J., Lam, J., Graves, E., Wu, W. (2009) Radar remote monitoring of vital signs. IEEE Microwave Magazine, 10(1), pp. 47-56.

Li, C., Lin, J. (2010) Recent Advances in Doppler Radar Sensors for Pervasive Healthcare Monitoring. Proceedings of Asia-Pacific Microwave Conference 2010, pp.283-290.

Lin, J. C. (1992) Microwave sensing of physiological movement and volume change: A review. Bioelectromagnetics, 13, pp.557-565.

Matsui, T., Hakozaki, Y., Suzuki, S., Usui, T., Kato, T., Hasegawa, K., Sugiyama, Y., Sugamata, M., Abe, S. (2010) A novel screening method for influenza patients using a newly developed non-contact screening system, Journal of Infection, 60(4), pp.271-277.

Matsui, T., Suzuki, S., Ujikawa, K., Usui, T., Gotoh, S., Sugamata, M., Abe, S. (2009) Development of a non-contact screening system for rapid medical inspection at a quarantine depot using a laser Doppler blood-flow meter, microwave radar, and infrared thermography, Journal of Medical Engineering \& Technology, 33(6), pp.481-487.

Miyake, S. (2001) Multivariate workload evaluation combining physiological and subjective measures. Int J Psychophysiol 40, pp.233-238

Morris, L.G., Kleinberger, A., Lee, K.C., Liberatore, L.A., Burschtin, O. (2008) Rapid risk stratification for obstructive sleep apnea, based on snoring severity and body mass index.Otolaryngol Head Neck Surg, 139(5), pp.615-618.

Obeid, D., Issa, G., Sadek, S., Zaharia, G., El Zein, G. (2008) Low power microwave systems for heartbeat rate detection at 2.4, 5.8, 10 and $16 \mathrm{GHz}$. First International 
Symposium on Applied Sciences on Biomedical and Communication Technologies, pp.1-5.

Petkie, D.T., Benton, C., Bryan, E. (2009) Millimeter wave radar for remote measurement of vital signs. IEEE Radar Conference, pp.1-3.

Princi, T., Parco, S., Accardo, A., Radillo, O., DeSeta, F., Guaschino, S. (2005) Parametric evaluation of heart rate variability during the menstrual cycle in young women. Biomed Sci Instrum 41, pp.340-345.

Rowe, M.A., Kelly, A., Horne, C., Lane, S., Campbell, J., Lehman, B., Phipps, C., Keller, M., Benito, A.P. (2009) Reducing dangerous nighttime events in persons with dementia by using a nighttime monitoring system. Alzheimers Dement, 5(5), pp.419-426.

Saunders, W.K. (1990) CW and FM Radar. in Radar Handbook, 2nd ed. M.I.Skolnik) San Francisco, MaGraw-Hill, Inc. pp.14.1-14.45

Scientfic Committee on Emerging and Newly Identified Health Risk (SCENIHR) (2006) Possible effects of Electromagnetic Fields (EMF) on Human Health.

Singh, N., Mironov, D., Armstrong, P.W., Ross, A.M., Langer, A. (1996) Heart rate variability assessment early after acute myocardial infarction. Pathophysiological and prognostic correlates. Circulation, 93, pp. 1388-1395.

Sirevaag, E.J., Kramer, A.F., Wickens, C.D., Reisweber, M., Strayer, D.L., Grenell, J.F. (1993) Assessment of pilot performance and mental workload in rotary wing aircraft. Ergonomics 36, pp.1121-1140.

Suzuki, S., Matsui, T., Imuta, H., Uenoyama, M. (2008) A novel autonomic activation measurement method for stress monitoring: non-contact measurement of heart rate variability using a compact microwave radar. Medical \& Biological Engineering \& Computing, 46(7), pp. 709-714.

Suzuki, S., Matsui, T., Kawahara, H., Gotoh, S. (2009) Development of a Noncontact and Long-Term Respiration Monitoring System Using Microwave Radar for Hibernating Black Bear. Zoo Biology, 28(3), pp.259-270.

Suzuki, S., Matsui, T., Kawahara, H., Ichiki, H., Shimizu, J., Kondo, Y., Yura, H., Gotoh, S., Takase, B., Ishihara, M. (2009) A non-contact vital sign monitoring system for ambulances using dual-frequency microwave radars, Medical \& Biological Engineering \& Computing, 47(1), pp.101-105.

Suzuki, S., Matsui, T., Sugawara, K., Asao, T., Kotani, K. (2011) An approach to remote monitoring of heart rate variability (HRV) using microwave radar during a calculation task, Journal of Physiological Anthropology, 30(6), pp.241-249.

Tanaka, S., Matsumoto, Y., Wakimoto, K. (2002) Unconstrained and non-invasive measurement of heart-beat and respiration periods using a phonocardiographic sensor. Med Biol Eng Comput, 40(2), pp.246-252.

Vincent, A., Craik, F.I., Furedy, J.J. (1996) Relations among memory performance, mental workload and cardiovascular responses. Int J Psychophysiol 23, pp.181-98.

Wang, F., Tanaka, M., Chonan, S. (2006) Development of a wearable mental stress evaluation system using PVDF film sensor. J Adv Sci, 18, pp.170-173.

Watanabe, K., Watanabe, T., Watanabe, H., Ando, H., Ishikawam, T., Kobayashi, K. (2005) Noninvasive measurement of heartbeat, respiration, snoring and body movements 
of a subject in bed via a pneumatic method.IEEE Trans Biomed Eng, 52(12), pp.2100-2107.

Zhou, Q., Liu, J., Host-Madsen, A., Boric-Lubecke, O., Lubecke, V. (2006) Detection of multiple heartbeats using Doppler radar," IEEE ICASSP 2006 Proceedings, 2, pp.1160-1163. 


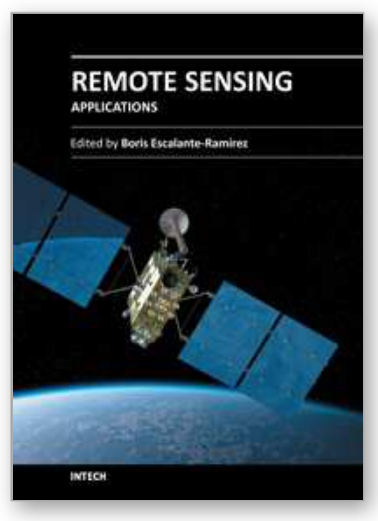

\author{
Remote Sensing - Applications \\ Edited by Dr. Boris Escalante
}

ISBN 978-953-51-0651-7

Hard cover, 516 pages

Publisher InTech

Published online 13, June, 2012

Published in print edition June, 2012

Nowadays it is hard to find areas of human activity and development that have not profited from or contributed to remote sensing. Natural, physical and social activities find in remote sensing a common ground for interaction and development. This book intends to show the reader how remote sensing impacts other areas of science, technology, and human activity, by displaying a selected number of high quality contributions dealing with different remote sensing applications.

\title{
How to reference
}

In order to correctly reference this scholarly work, feel free to copy and paste the following:

Satoshi Suzuki and Takemi Matsui (2012). Remote Sensing for Medical and Health Care Applications, Remote Sensing - Applications, Dr. Boris Escalante (Ed.), ISBN: 978-953-51-0651-7, InTech, Available from: http://www.intechopen.com/books/remote-sensing-applications/remote-sensing-for-medical-and-healthcareapplications

\section{INTECH}

open science | open minds

\section{InTech Europe}

University Campus STeP Ri

Slavka Krautzeka 83/A

51000 Rijeka, Croatia

Phone: +385 (51) 770447

Fax: +385 (51) 686166

www.intechopen.com

\section{InTech China}

Unit 405, Office Block, Hotel Equatorial Shanghai

No.65, Yan An Road (West), Shanghai, 200040, China

中国上海市延安西路65号上海国际贵都大饭店办公楼 405 单元

Phone: +86-21-62489820

Fax: $+86-21-62489821$ 
(C) 2012 The Author(s). Licensee IntechOpen. This is an open access article distributed under the terms of the Creative Commons Attribution 3.0 License, which permits unrestricted use, distribution, and reproduction in any medium, provided the original work is properly cited. 